\title{
Henle Fibre Layer Haemorrhage after a Valsalva Manoeuvre
}

\author{
Christoph Leisser $^{\mathrm{a}} \quad$ Josef Christian Huemer ${ }^{\mathrm{a}} \mathrm{b} \quad$ Oliver Findl $^{\mathrm{a}}$ \\ aVIROS - Vienna Institute for Research in Ocular Surgery, A Karl Landsteiner Institute, \\ Hanusch Hospital, Vienna, Austria; ${ }^{b} \mathrm{NIHR}$ Biomedical Research Center at Moorfields Eye \\ Hospital NHS Foundation Trust and UCL Institute of Ophthalmology, London, UK
}

\section{Keywords}

Henle fibre layer haemorrhage $\cdot$ Intravitreal application of recombinant tissue plasminogen activator · Valsalva bleeding

\begin{abstract}
A male patient, 59 years of age, presented with sudden deterioration of visual acuity (VA) caused by a Henle fibre layer haemorrhage (HFLH) in our outpatient department. He reported being under treatment with acetylsalicylic acid and experienced a Valsalva manoeuvre before he observed reduced vision. Due to the impairment caused by the haemorrhage, disturbing the patient's daily activities, intravitreal injection of recombinant tissue plasminogen activator combined with SF6 and bevacizumab was offered. After this procedure, the HFLH disappeared, VA improved from 20/40 to 20/20 (Snellen) 3 months after treatment and a typical hyperreflective vertical line was seen in optical coherence tomography.
\end{abstract}

(C) 2021 The Author(s).

Published by S. Karger AG, Basel

\section{Introduction}

Henle fibre layer haemorrhage (HFLH) as a descriptive feature in optical coherence tomography (OCT) [1-3], was shown to be associated with macular telangiectasia type 2 in earlier reports [1, 2]. A recent publication reported HFLH to be associated with increased systemic central venous pressure (due to intracranial haemorrhage caused by trauma or aneurysm, trunk trauma, general anaesthesia, Valsalva bleeding, epidural anaesthesia, and hypertension), local retinal venous pressure (caused by branch or central retinal vein occlusion, pars plana vitrectomy, decompression retinopathy, and local eye trauma), or local pathologies affecting the deep capillary plexus (such as polypoidal choroidal vasculopathy, macular telangiectasia type 2, myopic degeneration, and age-related macular degeneration) [3]. 

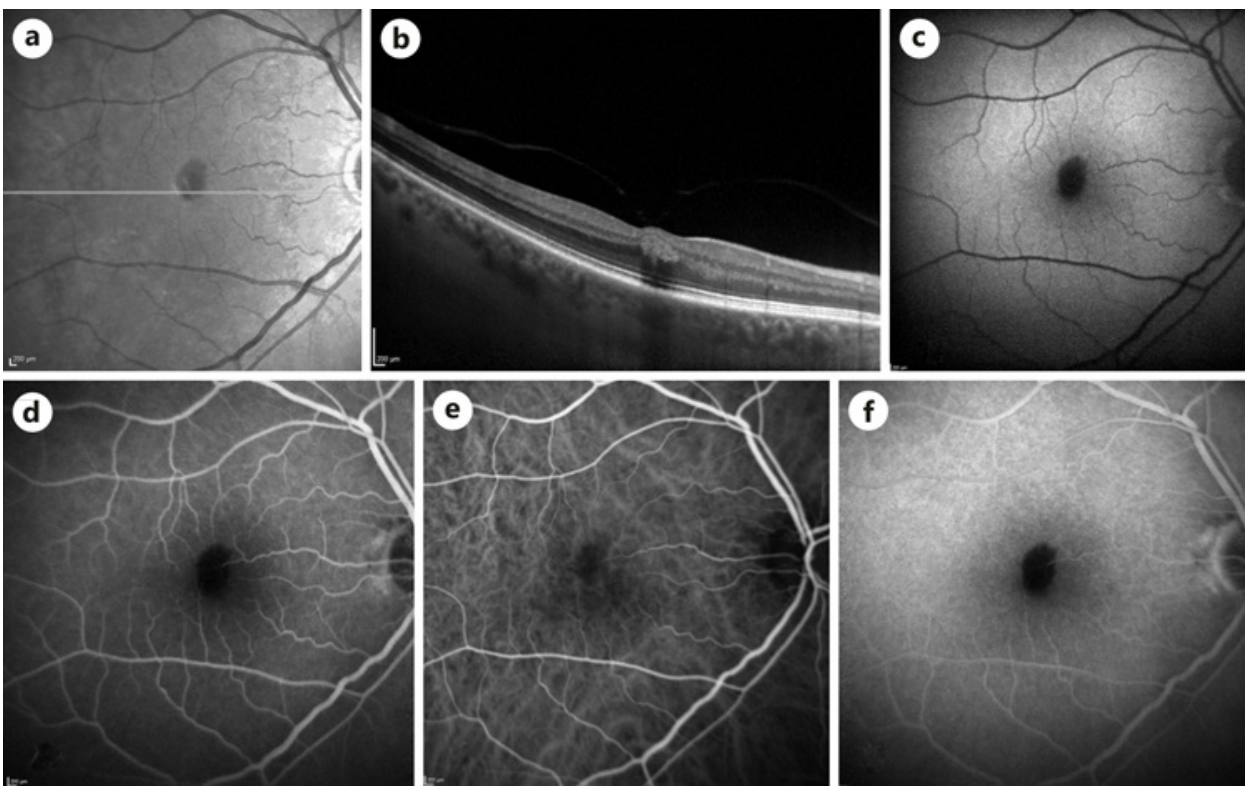

Fig. 1. Montage of multimodal imaging at first visit - near infrared reflectance (a) shows a hyporeflectant oval lesion, corresponding to a HFLH (appearing as hyperreflective lesion in the OCT) in the enhanced depth image OCT (b). Fundus autofluorescence (c), fluorescein angiography (d), early and late indocyanine angiography (e, f) show no signs of vascular pathology or macular neovascularization. HFLH, Henle fibre layer haemorrhage; OCT, optical coherence tomography; MNV, macular neovascularization.

HFLH typically presents with a petaloid pattern of haemorrhage, located in the Henle layer of the retina. The deep capillary plexus of the retina was recently reported to be the predominant part of the retina with respect to venous outflow [4, 5], indicating a possible reason for the location of haemorrhages in the Henle layer. Herein, we present a case of a Valsalva manoeuvre associated HFLH, successfully treated with intravitreal anti-vascular endothelial growth factor injection, recombinant tissue plasminogen activator (rTPA) and pneumatic displacement with SF6 gas tamponade.

\section{Case Report}

A male patient, 59 years of age, presented with a sudden deterioration of visual acuity (VA) to his right eye at our outpatient department. His medical history included the daily intake of $100 \mathrm{mg}$ of acetylsalicylic acid due to cardiovascular risk factors in the last 11 months. Cardiovascular risk factors included arterial hypertension, hypercholesterinemia, and presence of episodic angina pectoris. Before the reduction in VA, he confirmed to have succumbed a Valsalva manoeuvre, by heavy pressing for defecation, and careful questioning did not reveal any other reasons, such as trauma, infection, or systemic disease, leaving the Valsalva manoeuvre as the most likely cause.

On clinical examination, the VA of the affected eye was 20/40 Snellen. Retinal fundus examination revealed a solitary foveal intraretinal haemorrhage, with no additional signs of retinal or vitreous affection. No signs of retinal disease of any kind were observed in the fellow eye. To investigate the origin of the haemorrhage, we conducted multimodal imaging including an OCT with enhanced depth imaging, OCT angiography (OCTA), fluorescein, and 

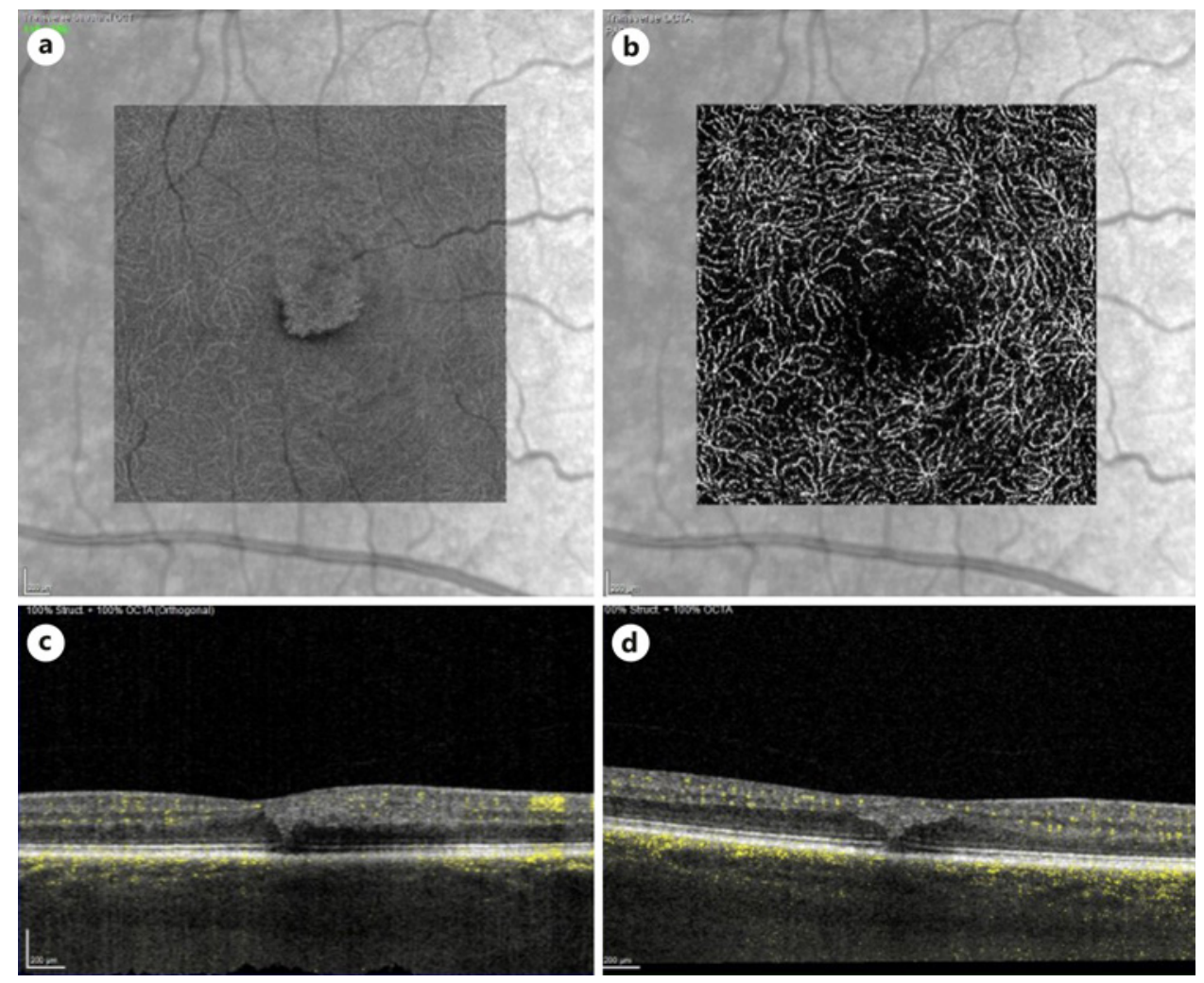

Fig. 2. OCTA at first visit - enface optical coherence angiography (a) and OCTA slab of deep capillary plexus (b) with according OCTA b-scans (c, d) displaying the HFLH with underlying shadowing artefact. HFLH, Henle fibre layer haemorrhage; OCTA, optical coherence tomography angiography; OCT, optical coherence tomography.
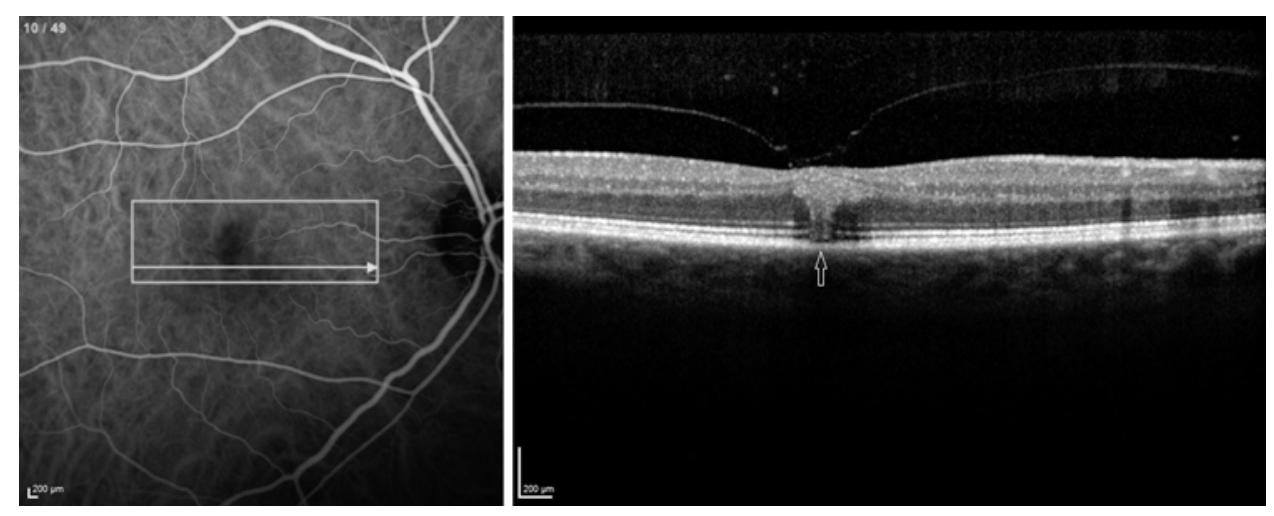

Fig. 3. OCT at first presentation - HFLH shows a discrete involvement of the ellipsoid zone. HFLH, Henle fibre layer haemorrhage; OCT, optical coherence tomography.

indocyanine green angiography (Fig. 1, 2). Since the decreased VA severely affected the patient's daily activities and the discrete involvement of the ellipsoid zone in the haemorrhage (Fig. 3), intravitreal injection of $0.1 \mathrm{~mL}$ rTPA $(50 \mu \mathrm{g}$ ) combined with $0.2 \mathrm{~mL}$ of $100 \%$ SF6 and $0.05 \mathrm{~mL}$ bevacizumab was offered to the patient 1 day after occurrence of the HFLH. An anterior chamber paracentesis was conducted at the end of the procedure to establish normal levels of IOP. Postsurgical topical therapy included brinzolamide eye drops for IOP 


\section{Case Reports in Ophthalmology}
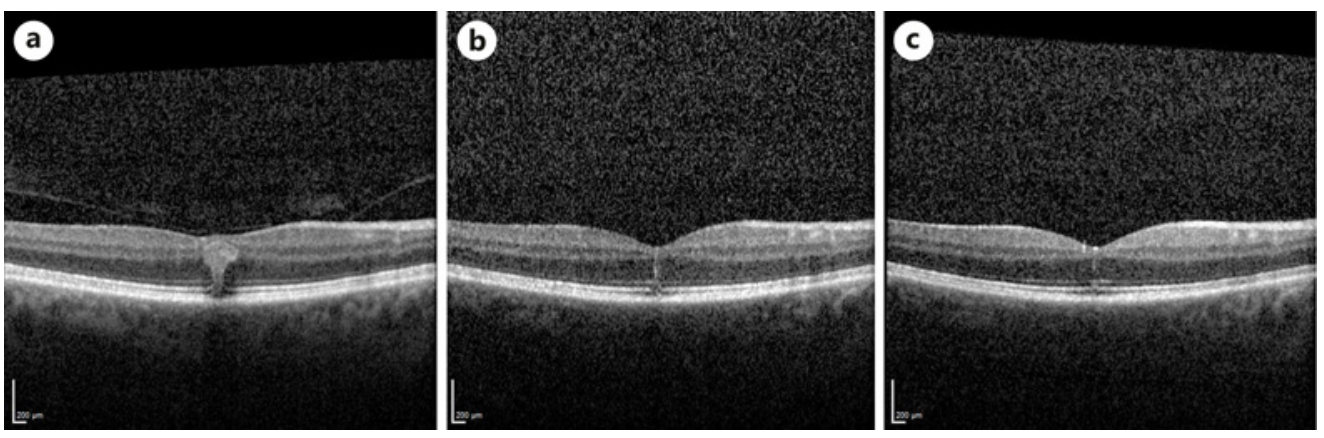

Fig. 4. OCT follow-up - OCT scans 1 day after surgery (a) with already diminished HFLH, absorption at month one (b) and restoration of the ellipsoid zone 8 weeks after surgery (c). HFLH, Henle fibre layer haemorrhage; OCT, optical coherence tomography.

control, as IOP was elevated 1 day after surgery but could be decreased to normal levels with topical therapy, and bromfenac and ofloxacin eye drops as routine postsurgical therapy for the first postsurgical week. At the follow-up examination 1 month after surgery, the HFLH was no longer present, leaving a typical hyperreflective vertical line in OCT (Fig. 4), and VA was improved from 20/40 to 20/25 Snellen 2 months and 20/20 Snellen 3 months after surgery.

\section{Discussion}

HFLH was reported to show spontaneous resorption within 3 months, in some cases leaving ellipsoid zone disruption or a hyperreflective vertical line, that can be observed with OCT [3]. As in our case, HFLH led to decreased VA affecting daily life activities and haemorrhage involved the ellipsoid zone (Fig. 3), we offered the patient intravitreal rTPA, combined with SF6 and bevacizumab.

Use of bevacizumab could be controversially discussed in this indication, especially as we did not find signs for polypoidal choroidal vasculopathy or macular neovascularization (MNV) at the time of first presentation, but a discrete involvement of the ellipsoid zone in the haemorrhage. Nevertheless, macular haemorrhages, in clinical practice are often followed by the discovery of MNV after resorption of the haemorrhage and, therefore, were offered to our patient in a prophylactic manner. No additional intravitreal injections of bevacizumab were given, due to the fact, that we found a typical vertical hyperreflective line in the postoperative OCTs, without any additional signs of MNV.

Haemorrhages after a Valsalva manoeuvre are mainly reported to be located under the internal limiting membrane [6,7], and therefore, laser membranotomy is recommended when the haemorrhage has not regressed within 3 weeks [7]. Location of a Valsalva-related haemorrhage in the Henle layer appears to be rare and needs a different way of treatment, as laser membranotomy would result in tissue damage at the fovea. While HFLHs usually regresses within 3 months [3], patients with vision loss affecting daily life activities, as in our case, prefer therapeutic options to quickly restore their VA. Intravitreal injection of rTPA and SF6 appears to be a safe and successful option in such cases.

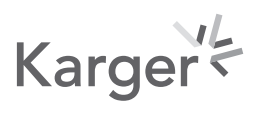


Leisser et al.: Henle Fibre Layer Haemorrhage

\section{Statement of Ethics}

Written informed consent was obtained from the patient to publish the case.

\section{Conflict of Interest Statement}

O. Findl is a scientific advisor for Alcon, Croma, Carl Zeiss Meditec AG, and Merck, N. All the authors declare that there are no conflicts of interest.

\section{Funding Sources}

There was no funding.

\section{Author Contributions}

C. Leisser ${ }^{1-3}$, J. Huemer ${ }^{1,2,4}$, and O. Findl ${ }^{1,4}$ : (1) conception of the study, (2) data acquisition, (3) preparation of the manuscript, and (4) critical review of the manuscript. All the authors state that they approve the final version of the manuscript for publication.

\section{References}

1 Rahimy E, Vander J. I see a seashell in my right eye. JAMA Ophthalmol. 2014;132(12):1413.

2 Au A, Hou K, Baumal CR, Sarraf D. Radial hemorrhage in henle layer in macular telangiectasia type 2. JAMA Ophthalmol. 2018;136(10):1182-5.

3 Baumal CR, Sarraf D, Bryant T, Gui W, Muakkassa N, Pichi F, et al. Henle fibre layer haemorrhage: clinical features and pathogenesis. Br J Ophthalmol. 2020;bjophthalmol.

4 Garrity ST, Paques M, Gaudric A, Freund KB, Sarraf D. Considerations in the understanding of venous outflow in the retinal capillary plexus. Retina. 2017;37(10):1809-12.

5 Freund KB, Sarraf D, Leong BCS, Garrity ST, Vupparaboina KK, Dansingani KK. Association of optical coherence tomography angiography of collaterals in retinal vein occlusion with major venous outflow through the deep vascular complex. JAMA Ophthalmol. 2018;136(11):1262-70.

6 Duane TD. Valsalva hemorrhagic retinopathy. Trans Am Ophthalmol Soc. 1972;70:298-313.

7 Matonti F, Donadieu V, Hoffart L, Dornadin A, Nadeau S, Roux S, et al. Early treatment with Nd: YAG laser for Valsalva retinopathy: results of five cases and literature review. J Fr Ophthalmol. 2013;36(7):604-9. 